\title{
Los patrones de crianza de la niñez en Nicaragua: un balance de los estudios realizados en el país
}

\author{
Luis Serra Vásquez \\ Investigador del Centro de Análisis Socio-Cultural (CASC), Universidad Centroamericana. E-mail: lserra@ns.uca.edu.ni
}

\section{Recibido: enero de 2007 / Aceptado: julio de 2007}

ESTE ARTículo ABORDA El TEMA DE LOS PATRONES DE CRIANZA de la niñez en el ámbito familiar y está basado en una revisión bibliográfica de los estudios realizados durante la última década en Nicaragua. Gran parte son diagnósticos rápidos en ámbitos locales con familias pobres de sectores urbanos, realizados con el fin de conocer la problemática de la crianza infantil y contribuir a una posterior intervención social. Otra parte consiste en estudios basados en encuestas realizadas en algunos municipios urbanos y rurales seleccionados, y sólo en dos casos con una muestra representativa a nivel nacional. Queda pendiente realizar estudios interdisciplinarios, explicativos y comparativos entre estilos de crianza de distintos grupos socio-culturales del país.

Los estudios seleccionados muestran que en Nicaragua existe una ambivalencia en la vida familiar y estilos de crianza. Por un lado, la familia constituye la principal organización de supervivencia material y afectiva basada en la ayuda recíproca que se establece entre parientes; por otro lado, representa un espacio de socialización de relaciones de poder y de violencia que fomentan actitudes de dominación y de sumisión. El hogar puede convertirse en un espacio de injusticia, particularmente contra las mujeres y las niñas/os, obstaculizando un desarrollo humano integral. Para enfrentar esta problemática, no es suficiente la aprobación de leyes, éstas deben de complementarse con acciones dirigidas al cambio de patrones culturales arraigados en el imaginario colectivo.

Palabras clave: crianza de niños-Nicaragua / educación de niños-investigaciones / educación en el hogar / relaciones de familia

\section{Introducción}

Este artículo aborda el tema de los patrones de crianza de la niñez en el ámbito familiar. Está basado en una revisión bibliográfica de los estudios realizados en la última década en Nicaragua. Forma parte de un estudio comparativo a nivel latinoamericano que dirige en nuestro país Manuel Ortega bajo la coordinación regional del instituto PROMUNDO de Brasil con la cooperación de Save the Children Noruega y Suecia. 
Nuestro estudio revela que la mitad de las investigaciones encontradas son diagnósticos rápidos en ámbitos locales con familias pobres de sectores urbanos, realizados con el fin de conocer la problemática de la crianza infantil y contribuir a una posterior intervención social. Otra parte consiste en estudios basados en encuestas realizadas en algunos municipios urbanos y rurales seleccionados, y sólo en dos casos con una muestra representativa a nivel nacional.

En cuanto al enfoque, predominan los abordajes desde la psicología, sociología y ciencias jurídicas. Estas disciplinas hacen uso y a veces combinación de una diversidad metodológica que incluye métodos cualitativos y cuantitativos. De esta manera, la mayoría de los estudios logran rescatar la visión y la voz de los niños/as, especialmente su valoración de la familia, sus relaciones con padres/madres y otros miembros, así como sus aspiraciones al futuro. En algunos estudios realizados con un enfoque de derechos humanos, se analiza el marco jurídico legal establecido en Nicaragua y su vigencia en la realidad social. La mayoría plantea propuestas de políticas y acciones para mejorar la calidad de vida de la niñez. Lamentablemente, muchos de ellos no han sido publicados y su circulación es reducida.

En gran parte de las investigaciones realizadas, el tema de los estilos de crianza de los niños y niñas suele ser abordado de forma breve por privilegiar una diversidad de temas (salud, educación, derechos humanos, relaciones de género) y de espacios sociales (familia, escuela, comunidad, trabajo), lo que no ha facilitado un análisis en profundidad de los estilos de crianza en los hogares y sus factores determinantes.

Los estudios realizados hasta la fecha constituyen un avance en el conocimiento de la situación de crianza en el país y plantean diversas hipótesis explicativas a verificar en posteriores estudios. Sin embargo, el carácter predominantemente exploratorio y descriptivo de la mayoría de ellos no permite profundizar en el análisis explicativo o comparativo de estos estudios, quedando pendiente este reto de investigación interdisciplinaria y participativa. A continuación se exponen, de manera más detallada, los resultados de la revisión bibliográfica que son relevantes para nuestro estudio

\section{Perfil de la niñez y adolescencia en Nicaragua}

La estructura demográfica de Nicaragua se presenta como una pirámide de base ancha, donde los menores de 15 años constituyen el 37.3\% de la sociedad en una población total de 5,142.098 habitantes según el Censo Nacional de 2005, con una tasa de fecundidad de 3.2 hijos por mujer y una tasa de crecimiento que ha bajado sorprendentemente en la ultima década (1995-2005) a 1.7\% anual (INEC, 2006). Se estima que del millón de emigrantes nicaragüenses hacia Costa Rica y Estados Unidos, el 40\% son niños/as y adolescentes.

Año tras año los adolescentes se incorporan crecientemente al grupo adulto en edad productiva (15-64 años), mientras la población mayor de 65 continúa siendo relativamente reducida. Este fenómeno, conocido como "bono demográfico", representa un capital humano importante para el desarrollo futuro del país en términos económicos y sociales, sólo que a condición de efectuar inversiones en educación, salud y empleo para estos niños/as y adolescentes, aspecto en el que Nicaragua ha sido deficitaria. 
Según el Informe de Desarrollo Humano, Nicaragua se ubica en un nivel medio bajo con un índice de 69 en 2005, en la posición 112 de 175 países del mundo, siendo el tercer país más bajo del continente (PNUD, 2005). Según el método de medición por "agregado de consumo", casi la mitad de la población nicaragüense (46.2\%) se encuentra en condiciones de pobreza, dentro de este grupo, un 14.9 \% vive en situación de extrema pobreza (INEC, 2005). Otros estudios basados en el enfoque de "necesidades básicas insatisfechas" muestran niveles mayores de pobreza. Los niños/as, especialmente los menores de 5 años, representan uno de los grupos sociales fuertemente afectados por la pobreza, pues 6 de cada 10 niños están en situación de pobreza y 2 de ellos/as en extrema pobreza. La situación de pobreza, la emigración, la violencia interpersonal y la paternidad irresponsable contribuyen a la desintegración familiar y por ende a la desatención de la niñez.

En los últimos años, unas 800,000 niñas, niños y adolescentes han quedado excluidos del sistema educativo formal, un 19\% a nivel primario, un 63\% en secundaria y un 88\% a nivel universitario. De los niños que ingresan a primer grado, sólo el 25\% concluye la primaria. El analfabetismo alcanza al 32\% de la población en las ciudades y a un 52\% en zonas rurales. En materia de salud, la tasa de mortalidad infantil es de 31 por 100 nacidos vivos, el 25\% presenta desnutrición crónica y el 36\% no ha registrado su nacimiento en el registro civil (Amaya, 2004).

Paralelamente al empobrecimiento se observa un crecimiento del trabajo infantil en labores de riesgo como una estrategia familiar de supervivencia. La encuesta nacional de 2005 identifica a 238,827 niños/as en trabajos remunerados, lo que representaba un 13.4\% de la población estimada entre 5 y 17 años. Un 36\% tenía menos de 14 años, que es la edad legal mínima para trabajar, lo que muestra una violación de las leyes vigentes. Dos tercios de los niños, niñas y adolescentes trabajadores se ubicaban en el campo, mientras a nivel urbano se encontraba un tercio del total. Las consecuencias del trabajo infantil se observan en la deserción escolar o bajo rendimiento, en el estado nutricional y crecimiento, en las enfermedades crónicas y traumas psicológicos no visibles (MITRAB-OIT, 2007). Todo esto, que perjudica tanto su desarrollo personal como social, ocurre a pesar de que la Constitución establece que "[s]e prohíbe el trabajo de los menores, en labores que puedan afectar su desarrollo normal o su ciclo de instrucción obligatoria. Se protegerá a los niños y adolescentes contra cualquier clase de explotación económica y social” (art.84).

Según cifras oficiales, se estimaba que en Nicaragua había más de 600 mil niños, niñas y adolescentes que vivían en situaciones de riesgo por abandono, maltrato, trabajo infantil, violencia, abuso y explotación sexual comercial, adicción a drogas, e infracción de la ley penal (CONAPINA, 2001). La Coordinadora de ONG que Trabajan con la Niñez y Adolescencia considera que "por su peso poblacional y por la exclusión que históricamente han vivido, los/as niños/as y los adolescentes son los más empobrecidos del país..... La situación de vulnerabilidad que viven los/as niños/as y adolescentes los ha excluido socialmente y les imposibilita su desarrollo” (Chamorro \& Gutiérrez, 2001:8).

En la práctica, las oportunidades que brinda la sociedad nicaragüense a los niños/as y adolescentes se diferencian de acuerdo a las capacidades económicas, sociales y afectivas de las familias. Los niños/as de clase alta y media disfrutan de condiciones satisfactorias, 
mientras que la mayoría de los que viven en situación de pobreza goza de oportunidades muy limitadas para su desarrollo personal y social, ya que el Estado invierte muy escasamente en programas para favorecer el desarrollo de la niñez y la juventud.

\section{Marco legal y políticas de la niñez y adolescencia}

En los últimos años en Nicaragua se han experimentado avances importantes en el ámbito de la legislación y las políticas públicas en beneficio de la niñez y la adolescencia. En ellas se introducen nuevos enfoques que buscan superar la concepción del niño como sujeto pasivo de protección, compasión o represión, que debe subordinarse a la voluntad de los adultos dada su calidad de "menor", es decir, de ser humano de segunda categoría, carente de derechos y oportunidades.

- El gobierno ha ratificado la Convención de las Naciones Unidas sobre los Derechos del Niño (1990). Fue incorporada en las reformas a la Constitución Política (1995) y en Protocolos Facultativos de la Convención sobre los derechos de Niños en Conflictos Armados, la Venta y Prostitución Infantil.

- El país ha ratificado el Convenio 182 de la OIT sobre la eliminación de las peores formas de trabajo infantil, además del Convenio 138 relativo a la edad mínima de admisión de empleo (14 años). Éstos han sido recogidos en el nuevo Código Laboral que contiene un capítulo especial referido al trabajo de niños/as y jóvenes.

- Se ha aprobado la Ley para la Prevención y Sanción de la Violencia Intrafamiliar (1996) y la Ley sobre Delitos Sexuales (2001), así como reformas al Código Penal actualmente en la Asamblea Nacional.

- Se ha aprobado un Código de la Niñez y la Adolescencia (1998) y una Ley de Promoción del Desarrollo Integral de la Juventud (2001).

- Se han aprobado leyes de familia como la Ley de Alimentos; Ley Reguladora de las Relaciones Madre, Padre, Hijos; Ley de Adopción; y se encuentra en elaboración un Código de Familia.

- Se creó el Consejo Nacional de Protección y Atención Integral a la Niñez y Adolescencia, adscrito a la Presidencia como órgano rector para la promoción de estos derechos (2001). También se ha creado la Comisión de Lucha contra la Violencia hacia la Mujer, Niñez y Adolescencia, y la Secretaria de la Juventud.

- Se ha elaborado una Política Nacional de Protección Integral de la Niñez y Adolescencia, un Plan de Acción Nacional para la Niñez y Adolescencia (2002-2011), un Plan de Desarrollo Integral para la Prevención de la Violencia Juvenil y un Plan Nacional para la Prevención y Erradicación del Trabajo Infantil y Protección de Adolescentes Trabajadores.

- Se instaló la Sub-Procuraduría Especial de la Niñez y Adolescencia (2000) dentro de la Procuraduría de Derechos Humanos y Comisarías de la Mujer y Niñez en 25 ciudades del país. 
- Se han conformado Comisiones Municipales Inter-institucionales para promover los derechos de la niñez en la mitad de los 151 municipios del país.

En general estas legislaciones y políticas adoptadas consideran al niño/a como sujeto de derechos, incluyendo el derecho a la protección especial y el derecho a condiciones de vida que permitan su desarrollo integral. Sin embargo, una encuesta a 1,200 niños/as y adolescentes muestra que sólo el 20.3\% dice conocer el Código de la Niñez de forma general, la mitad considera que no se está cumpliendo y solamente un $17.5 \%$ conoce la existencia de la Procuraduría Especial de la Niñez y Adolescencia (PDH, 2002).

Un obstáculo para el efectivo cumplimiento de los derechos de la niñez y adolescencia es la contradicción entre la política económica y la política social. Los gobiernos recientes "neoliberales", condicionados por los planes de ajuste acordados por el FMI, han reducido la inversión social y privatizado los servicios de salud y educación, afectando los derechos de los niños/as y jóvenes. De ahí que recientemente el Comité de los Derechos del Niño de Naciones Unidas haya recomendado al Estado de Nicaragua garantizar el cumplimiento de los Derechos de la Niñez y la Adolescencia mediante una mayor inversión social.

Otras limitantes para la aplicación de estos instrumentos son: la falta de voluntad política de algunos sectores de poder, la escasa asignación de recursos, la falta de mecanismos ágiles para asegurar el pleno cumplimiento de los derechos de niños/as y adolescentes, y el predomino de rasgos culturales de tipo autoritario y adultista. A nivel jurídico, queda pendiente establecer los centros especiales para adolescentes, elaborar un código de familia, ratificar el convenio de La Haya sobre adopciones e incorporar el Convenio 182 de la OIT en la legislación laboral.

En los 90 se desarrollaron diversas iniciativas a nivel local de formación y organización de niños/as y jóvenes para ampliar sus capacidades y oportunidades de superación. También surgieron experiencias innovadoras de participación de niños/as y adolescentes, tales como:

- La discusión de las propuestas del Código de la Niñez y su posterior divulgación, donde participaron más de 2,000 niños/as y jóvenes.

- La participación en diversos proyectos de desarrollo y la conformación de múltiples organizaciones de niños/as y jóvenes con apoyo de ONG, cooperación internacional y Movimiento Alcaldes Amigos de los niños/as. Se destacan el Movimiento de Niños/as y Adolescentes Trabajadores (NATRAS) y el Consejo Nacional de la Juventud.

- La formación de Gobiernos Estudiantiles en muchos centros educativos de primaria y secundaria como una instancia de participación junto con los padres, docentes y dirección, conforme la Ley de Participación Educativa (2002).

- Diversos estudios sobre la situación de la niñez, elaborados con metodologías participativas a nivel nacional y a nivel local, como la metodología "Niño a Niño".

- Programas educativos para y con niños/as y jóvenes (Ej. Los Cumiches, Tiempos de Ternura, Tita Ternura, Sexto Sentido). 


\section{Enfoques teóricos utilizados en los estudios sobre crianza}

La mayor parte de los estudios sobre crianza que hemos encontrado parten teóricamente del concepto de socialización como un proceso histórico de transmisión de la cultura, entendida ésta como los patrones de conducta, conocimientos, creencias, moral y costumbres que caracterizan una sociedad. La familia es concebida como "principal agente socializador" que forma la "personalidad socio-cultural de los individuos", incluyendo las relaciones de género y generacionales. El proceso de socialización de los niños/as adquiere características particulares de acuerdo a la edad o etapa de desarrollo psico-físico que atraviesan.

La familia, en tanto sistema de autoridad, constituye un orden productor de reglas que regulan las funciones, prácticas, relaciones y concepciones entre sus miembros, es decir, lo que significa ser madre, padre, hijo, hija, hermano, hermana, abuelo o tío. En el ámbito familiar se establecen relaciones de solidaridad y comunicación, pero también de oposición y conflicto entre sus miembros, dependiendo del sistema de autoridad que se establece y que constituye un elemento central de la familia (Castillo y Serra, 2003).

De igual modo, la crianza de hijos se ha definido como un proceso de socialización primaria donde "se crean y recrean relaciones sociales de autoridad, solidaridad y conflicto" (Agudelo et al., 2000:5). La crianza se concibe como un proceso continuo y complejo de desarrollo de las capacidades de los niños conforme a su edad y a su medio socio-cultural (Corea Tórrez et al., 2000). Zúñiga y Pineda (1997:3) entienden por crianza "los procesos de cuidado y protección, relaciones afectivas, socialización, enculturación y educación que los adultos les otorgan a los niños/as”. Se trata de un proceso multideterminado que abarca componentes ideacionales (creencias, saberes, valores) y prácticos (acciones habituales). Ambos elementos interrelacionados configuran "sistemas de crianza" condicionados por la cultura hegemónica.

Varios autores consideran que las percepciones de la realidad que tienen los niños/as son determinadas por las relaciones sociales en que están inmersos en el proceso de socialización primaria. Sin embargo, algunos estudios plantean que la socialización de los niños/as no es un proceso unidireccional de los adultos hacia los infantes sino de interrelación, ya que éstos/as son un sujeto que influye en el entorno proyectando sus acciones, intenciones y afectos. Lo contrario supone negar la capacidad de los niños/as y adolescentes para actuar de forma activa y determinante en la sociedad desempeñando un papel significativo en su propio desarrollo, en su familia y en la transformación de su comunidad (Castillo \& Serra, 2003).

La mayoría de los autores/as destaca el rol de la familia en la reproducción de las relaciones (desiguales) de género y la mistificación de la maternidad. Para CINCO, el análisis de los estilos de crianza implica el abordaje de las mentalidades, las relaciones de intercambio y poder, la organización de la vida cotidiana, la afectividad, la solidaridad, los conflictos (Agudelo et al., 2000).

Casi todos los estudios abordan las percepciones de los niños/as y adultos, consideradas como una construcción social que permite la interpretación de la realidad y guía el 
comportamiento humano. La subjetividad es entendida como las formas propias que tienen los seres humanos de ver el mundo y a sí mismos; y en el caso de los niños/as son propias y distintas a las de los adultos (Pineda \& Guerra, 1997). La noción de representación se refiere a las ideas, conceptos, imágenes, conocimientos, valoraciones y emociones que los seres humanos producen en su interacción con la realidad natural y social (Castillo \& Serra, 2003). En algunos estudios se retoma la teoría de género para comprender las diferencias culturalmente establecidas entre los roles de mujeres y hombres, incluyendo las funciones esperadas socialmente del padre y la madre en las relaciones con sus hijos/as (Centeno \& Castillo, 2005; Agudelo et al., 2000; Castillo \& Serra, 2003).

Los autores seleccionados consideran que la niñez no es una realidad uniforme y universal, sino variable conforme a su mundo social y cultural. Algunos estudios tratan de identificar las diferencias en los patrones de crianza por razones de sexo/genero, de edad, de nivel socio-económico y de residencia urbana o rural. Sin embargo, queda pendiente profundizar el análisis comparativo de distintas categorías y situaciones de la niñez así como de estilos de crianza. Varios estudios presentan el marco jurídico legal que rige en Nicaragua sobre la niñez y adolescencia, así como las definiciones de niñez y adolescencia contempladas en esas normativas con sus contradicciones (PDH, 2002 y 2004)

Los estudios concuerdan en el predominio y vigencia de una cultura autoritaria, patriarcal y "adultista" que considera a la niñez como objeto de propiedad y protección, y que desconoce los derechos de participación de los niños/as como sujetos sociales. El "adultismo" se ha definido como un sistema de representaciones y prácticas sociales que se refuerzan mutuamente reproduciendo una desigual distribución de recursos y poder entre adultos y niños (Castillo \& Serra, 2003).

La participación ha sido definida como un derecho de los niños/as de ser parte activa en la toma de decisiones en los distintos espacios de su vida, lo cual implica el desarrollo de capacidades de conocer, comunicar y generar cambios (CODENI, 2003). Algunos estudios distinguen niveles de participación de los niños/as a nivel familiar conforme factores como la apertura a diálogo de padres/madres y adultos cercanos, la seguridad del entorno social, el desarrollo de las capacidades del propio niño/a y otras condiciones básicas como salud, buena alimentación y atención con cariño (Castillo \& Serra, 2003). Otros autores consideran que la participación de los niños/as dentro de la familia depende del tipo de hogar, que puede ser:

a) Democrático: valoran a los niños y les permiten explorar;

b) Dominante: no dan oportunidades de expresión y acción (CANTERA, 1995).

\section{La familia en Nicaragua}

En esta sección presentamos los resultados de los estudios seleccionados sobre la situación de la familia, su dinámica, y la valoración que realizan tanto los niños/as como los adultos entrevistados.

La familia ha sido definida como un sistema socio-cultural abierto conformado por individuos con lazos de parentesco consanguíneo o político (Zúñiga \& Pineda, 1997). A pesar de la crisis 
que atraviesa actualmente, la familia en Nicaragua es una red social clave en la vida de las personas. Como señala el Informe de Desarrollo Humano de Nicaragua: "Por un lado, constituye la unidad de organización cotidiana de la existencia social y económica de las personas, por el otro, es el centro de su vida afectiva" (PNUD, 2000:97). Se observa una ambivalencia de la familia, por un lado es un resguardo frente a los riesgos sociales del entorno; por otro lado, es un espacio de violencia familiar y vulnerabilidad para algunos miembros.

En la realidad actual nicaragüense, existe una diversidad de tipos de familia distintos de la familia "clásica" (nuclear biparental o completa) compuesta por padre, madre e hijos que comparten un hogar. Sólo la mitad de los hogares nicaragüenses corresponde a este modelo nuclear, mientras que casi un tercio de los hogares es de tipo extenso compuesto por parientes de varias generaciones. Los hogares cuya jefa es mujer (modelo nuclear incompleto o monoparental) representan una cuarta parte a nivel nacional y un tercio en las ciudades (PNUD, 2000).

Según la Encuesta Nicaragüense de Demografía y Salud, más de la mitad (61.5\%) de los menores de 15 años vivía con ambos padres, $23 \%$ vivía con la madre, un $3 \%$ con el padre y $10.3 \%$ con ninguno de los dos, que sumaban un total de 220.000 en el año 2000. En el ámbito rural, la convivencia con ambos padres era mayor (69.5\%) que en el ámbito urbano (54\%), donde un tercio de los niños/as vivía sólo con la madre (31.5\%.) quien debía de asumir la jefatura del hogar debido al abandono paterno, viudez, violencia o desarraigo (INEC, 2002). No obstante la ausencia paterna, los hombres reclaman su derecho a ser la máxima autoridad en la familia, según muestra un estudio realizado a nivel nacional donde dos tercios de hombres entrevistados dijeron que "el hombre debe ser siempre el jefe del hogar" y quien debe de tener la última palabra en las decisiones familiares (Centeno \& Castillo, 2005:95).

Condicionados por la pobreza y la migración, en muchos hogares nicaragüenses se observa que conviven en una misma vivienda un complejo de familias nucleares numerosas ligadas o no por lazos de parentesco, que establecen acuerdos para la convivencia, la crianza de los hijos y las actividades económicas de generación de ingresos (Fauné, 1995a). Los hogares en extrema pobreza tienen el doble de miembros que los hogares no pobres debido a una tasa de fecundidad elevada y a la estrategia de integrar a más miembros a fin de ampliar su base de ingresos familiares (PNUD, 2000).

En los estudios indicados encontramos que casi todos los niños/as valoran su familia como algo muy importante en sus vidas, mostrando así la relevancia de esta red social primaria en el desarrollo de la niñez. Una encuesta nacional con 1,200 niños/as señala que un 82\% de la niñez se siente alegre en su hogar, más en el área rural que urbana, mientras que un 14\% no se siente bien en su casa por motivos de ausencia de padre o madre, maltrato o pobreza (PDH, 2002).

Otro estudio realizado en 160 hogares de comunidades urbanas y rurales muestra que los niños/as piensan que sus familias son importantes por varias razones: "nos ayudan y cuidan"... "me dan lo que necesito"... "nos quieren"... "se preocupan de mi salud y educación"... "me protegen". Cuando les preguntaron a los niños/as ¿Crees que sos 
importante para tu familia?, casi todos (95\%) respondieron en forma afirmativa. La percepción de la mayoría de los niños/as sobre su familia era positiva. Sin embargo, un 20\% de la niñez tiene una valoración negativa de su familia por varias razones: disputas entre familiares, no los dejan jugar, los golpean y los maltratan verbalmente (Castillo \& Serra, 2003:77-78).

Un estudio realizado con niños de Managua indica que para los niños/as, el criterio para considerar una persona como miembro de su familia es que "me quiere", "puedo contar con ellos" y "me lo ha dicho". Este estudio destaca que las valoraciones de la familia varían según el sector socio-económico y el género de la niñez, así encontramos que los niños/as de sectores medios (sólo estudian) valoran más positivamente la vida en su hogar que los niños/ as pobres que trabajan y viven en condiciones de hacinamiento, necesidades insatisfechas y tensiones interpersonales. En términos de género, las niñas sienten menos seguridad en su casa que los niños, lo que se vincularía con abusos sexuales y trabajos pesados (Pineda \& Guerra, 1997).

Por otro lado, observamos que en la vida familiar las cuestiones que mayor rechazo le provocan a niños/as entrevistados son el maltrato, el exceso de trabajo, los pleitos entre familiares y que no los tomen en cuenta en las decisiones. También perciben negativamente la pérdida o la enfermedad de padres/madres o familiares. Frente a estos eventos, los niños/ as se ponen tristes, tienen miedo, enojo, preocupación. Los momentos de mayor satisfacción para ellos/as son cuando hay armonía en la familia y se reúnen, los tratan bien, les dan apoyo, juegan con ellos, los toman en cuenta y les reconocen sus cualidades (Pineda \& Guerra, 1997).

Por su parte, los hombres coinciden en que "lo más importante en la vida es la familia", según nos muestra el estudio realizado a nivel nacional (1,200 varones mayores de 15 años). Sin embargo, muchos (41\%) piensan que "es natural que el hombre tenga hijos fuera del matrimonio", reflejando la concepción patriarcal tradicional que le permite al hombre tener dos o más relaciones de pareja (y familias) simultáneas (Centeno \& Castillo, 2005:84).

Casi todos los estudios han enfocado a familias de escasos recursos, mostrando que la situación de pobreza afecta de modo particular a los niños/as que no reciben una alimentación suficiente, carecen de los materiales y uniforme escolares, no tienen espacios adecuados en sus casas ni tiempo disponible (por los trabajos domésticos o ventas) para el cumplimiento de sus deberes escolares, la sana recreación y el descanso. Los estudios consultados revelan condiciones de hacinamiento, ya que la mayoría de las viviendas cuenta con un área mínima para la vida cotidiana de sus habitantes.

\section{Concepciones sobre la crianza de niños/as}

Algunos estudios abordan directamente el tema de la crianza de los niños/as. En la investigación de PAININ a nivel nacional, los padres/madres entrevistados consideraron que "criar bien" a sus hijos significa educarlos, atender sus necesidades materiales y darles afecto. Por el contrario, la concepción de "criar mal” a sus hijos la asociaron con permitirles malos hábitos, darles maltrato o abandonarlos (Corea Tórrez et al., 2000). 
La principal expectativa que tienen los padres/madres de 66 barrios populares de Managua con el futuro de sus hijos es que terminen sus estudios y tengan una carrera. Su temor principal es que "agarre un vicio" o se meta en una pandilla (Corea Tórrez et al., 2000). Otro estudio realizado en un barrio pobre de Managua encuentra que casi todos los/as adultos piensan que la crianza de los hijos es una labor compartida entre padre y madre, sin embargo, en la práctica vemos que las mujeres asumen exclusivamente tareas como el cuido, alimentación y tareas escolares (Zúñiga, 1997).

La mayoría de los padres/madres entrevistados en Ciudad Sandino expresó quela transmisión de valores y normas a sus hijos se basa en su propia experiencia, en menor medida obedece a orientaciones religiosas (CANTERA, 1995). Sin embargo, hay padres/madres conscientes de sus limitaciones para criar a sus hijos y con afán de superación desean aprender sobre sexualidad, métodos educativos de corrección, etapas del desarrollo, nutrición, drogas, y problemas de aprendizaje, a fin de tener mayores herramientas para criar a sus hijos (Zúñiga, 1997).

Los resultados de todos los estudios confirman el rol fundamental que desempeñan las mujeres-madres, tanto en hogares monoparentales como nucleares, en la crianza de los hijos y en la reproducción familiar. Contrariamente, el padre aparece como una "figura periférica" en la socialización primaria de los niños/as. Quedan evidentes las limitaciones en la comunicación hijos-padres/madres y el predominio de formas autoritarias de establecer las reglas del juego en el ámbito familiar (Agudelo et al., 2000).

Un estudio sobre el tema de la paternidad en Nicaragua nos señala que la problemática social de la irresponsabilidad paterna en la crianza de los hijos/as está vinculada a la promiscuidad sexual y al rechazo a métodos anticonceptivos que conduce a embarazos no deseados. Los hombres inician su vida sexual antes que las mujeres, establecen relaciones sin vínculos afectivos y tienen en promedio un mayor número de parejas que las mujeres. Asimismo, se ha observado que el abandono de los hijos es mayor en los padres que tienen conflictos en sus relaciones con la madre (Montoya, 2001).

La concepción de masculinidad y paternidad que tienen los hombres nos ayuda a comprender los estilos de crianza predominantes. Un estudio reciente realizado a nivel nacional (1,200 hombres) destaca que la mayoría de los hombres entrevistados (48.6\%) se ubica en una visión tradicional de superioridad masculina que en la práctica significa la subordinación de mujeres y niños/as y el abandono paterno del hogar. Esta concepción aparece claramente en hombres del sector rural con bajo nivel educativo. Por otro lado, un perfil de hombres (41.2\%) expresa un discurso de igualdad de géneros, de relaciones afectivas y responsables entre padre e hijos/as y de dar cierta autonomía a las mujeres. Este perfil se ubica en sectores urbanos con educación secundaria. Un tercer perfil (10\%) se ubica en posiciones de transición entre los dos anteriores (Centeno \& Castillo, 2005).

El perfil de paternidad que predomina (49\%) es el del padre proveedor económico y formador de los hijos, pero que no prodiga afecto y deja la crianza a la madre. Un segundo perfil de padres (38\%) muestra una mentalidad más abierta a compartir la crianza con las madres y brindar afecto a sus hijos, así como apoyo económico. Por último, el estudio muestra un 
tercer perfil (13\%) de varones en situación de transición entre los modelos anteriores. Las variantes en la crianza de niños y niñas difieren según el género, la clase social y la religión. Por ejemplo, las personas de creencia evangélica manifestaron que su modelo de crianza provenía de Dios Padre: "Él quiere a todos sus hijos por igual, sean malos o buenos....." (Centeno \& Castillo, 2005:96).

Al preguntarles sobre el objetivo de tener hijos, 40\% de los 400 padres/madres entrevistados en Managua por CINCO respondió "para formar una familia”, 27\% dijo "para que cuiden a los padres/madres”, 9\% expresó “para que ayuden a los padres/madres en el trabajo”. El estudio muestra que culturalmente la sexualidad está asociada a la reproducción, es decir, que la procreación aparece como necesaria y "natural" en los matrimonios o uniones de hecho, así como para legitimar la identidad masculina del padre y femenina de la madre (Agudelo et al., 2000).

Otro estudio realizado a nivel nacional (840 adolescentes y púberes) nos indica que esta concepción cultural "tradicional" sobre sexualidad y embarazo se encuentra entre adolescentes de familias pobres del campo, mientras que en jóvenes urbanos de sectores medios se halla una concepción "moderna" de "sexualidad relacional" separada de la reproducción biológica. Más de dos tercios de los 120 púberes de 10-14 años (72.5\%) está de acuerdo en que "lo más importante para toda mujer en la vida es ser madre”, reflejando una visión tradicional del rol sociocultural de las mujeres (Ortega et al., 2000:33).

\section{Comunicación y afecto}

Todos los estudios reflejan que las madres juegan un rol insustituible en la atención a los hijos/as tanto en el ámbito afectivo como de sus necesidades básicas. También hay que señalar el rol de los hermanos y hermanas en el cuido de sus hermanos/as pequeños y apoyo en tareas escolares.

Un estudio nacional sobre crianza de niños/as menores de 6 años, indica que las madres tienen una estrecha comunicación con sus hijos/as desde tierna edad y estimulan su desarrollo motor, los hábitos de higiene, el lenguaje y el aprendizaje vía juegos. También se observaron los siguientes aspectos negativos en la comunicación de padres/madres a hijos: resaltar sus fallas, hablar mal del padre o madre, compararlos con hermanos, crearles temores, maltratarlos verbal o físicamente (Zúñiga, 2000).

Otro estudio realizado con 120 niños/as de Managua muestra que el rol de la madre en la atención a los hijos/as disminuye en el grupo de 6-12 años y aumenta el rol de las hermanas mayores y las abuelas. Tanto las madres como los padres dicen brindarles afecto a sus hijos/as mediante caricias, abrazos, chineos y besos, en mayor medida a los menores de 6 años. La comunicación habitual entre padres/madres-hijos ocurre a la hora de la comida que comparte la mayoría (Zúñiga \& Pineda, 1997).

En el caso del diagnóstico hecho en Managua con 797 padres/madres e hijos, se señala que las principales actividades que comparten hijos y padres/madres es ver televisión, platicar, comer juntos y salir a pasear. Sin embargo, hay que señalar que la televisión no facilita la 
interacción entre ellos. Según los niños, las formas comunes que usan sus padres/madres para expresar su cariño son la expresión verbal, la compra de bienes, la protección, y los abrazos y besos (Agudelo et al., 2000).

La comunicación familiar se ve afectada por la situación de pobreza. En los hogares de escasos recursos, el tiempo que los padres/madres dedican a sus hijos está limitado por razones de trabajo y disponibilidad, destacándose una mayor presencia y comunicación con la madre. En el diagnóstico realizado en Ciudad Sandino, la mayoría de los niños expresa tener igual confianza con ambos, padre y madre, (45\%), en segundo lugar aparece una confianza mayor con la madre (31\%) y en menor medida con el padre (5\%) (CANTERA, 1995).

La observación de 160 hogares en 4 municipios ha mostrado que, en general, el estado emocional predominante es de tranquilidad y las relaciones son generalmente cordiales, de colaboración y respeto entre hijos/as y padres/madres. La mayor parte de las comunicaciones de adultos a niños consiste en peticiones o en ordenes de los adultos, así como regaños por actitudes que son reprobadas y para dar informaciones varias. (Castillo \& Serra, 2003). Esto se confirma en otro estudio realizado en Managua: al preguntarles a los padres/madres cómo le solicitan a sus hijos que hagan una tarea, la mayoría de ellos/as (54\%) dice que les pide por favor. Sin embargo, se observaron contradicciones en la práctica del hogar (Agudelo et al., 2000).

En cuanto a su autoestima, los niños/as entrevistados en un estudio local han mostrado poseer un nivel alto de autoestima. Ellos/as se consideran importantes, capaces, y queridos por sus familias; tienen expectativas positivas sobre su vida futura. Se observa en ellos/ as una gran capacidad de resiliencia, es decir, adaptación positiva a las dificultades del entorno familiar y comunal. La capacidad de análisis que tienen los niños/as, y más aún los adolescentes, sobre su vida personal y su entorno social, abordando con seriedad problemas nacionales como la pobreza y el desempleo, y planteando propuestas de superación a los gobernantes y actores sociales, ha sido revelada en varios estudios. Sobre su visión de futuro, la mayoría de los niños/as expresó su ideal de alcanzar una profesión u oficio y su optimismo de poder lograrlo a pesar de su situación de pobreza (Castillo \& Serra, 2003).

Otro estudio en Managua muestra una mayor autoestima entre los niños de clase media y los niños pobres que participan en proyectos educativos. Por el contrario, en niños pobres ajenos a proyectos se observan actitudes de impotencia y fatalismo frente a la cruda realidad que viven. En cuanto a las percepciones de las diferencias por sexo/género, dos tercios de los/las entrevistados/as valoran que ambos son iguales, pero un tercio considera que su sexo/género tiene mayores capacidades que el otro (Pineda \& Guerra, 1997).

Los hombres encuestados a nivel nacional por el CASC valoran muy positivamente el tener hijos, lo que da sentido a su vida, siendo una bendición de Dios y un fruto del amor. La visión de los hijos como seguro de vejez de sus padres/madres o como ayuda a la supervivencia se observa mayormente en sectores rurales y pobres. Casi todos los varones encuestados (98\%) dicen que "hay que apoyar y cuidar a los hijos" aunque tengan problemas económicos, o ya no viva el padre en pareja con la madre (97\%). Igual consenso (96\%) encontramos en que 
"los padres/madres deben asumir la crianza de los hijos en ausencia de la madre" (Centeno \& Castillo, 2005:95).

En el estudio realizado a 708 personas de 6 municipios del país sobre migración de padres/ madres, vemos que si es el padre quien sale fuera del país, los niños quedan al cuido de su madre. Pero en caso de migrar la madre, los niños/as quedan al cuido de las abuelas o tías, ya que los papás no asumen su responsabilidad. La ausencia de sus padres/madres afecta el desarrollo de niños/as que sienten tristeza, soledad, desprotección, falta de cariño. Sin embargo, muchos hijos/as se comunican telefónicamente con sus padres/madres con bastante frecuencia, el tema más platicado es sobre la escuela. Sin embargo, 25\% tiene más de dos años de no verlos (PDH, 2004).

Un estudio reciente señala que la participación infantil en el ámbito familiar no ha sido promovida directamente por instituciones estatales o civiles en Nicaragua, aunque algunas ONG desarrollan acciones con madres y padres para promover los derechos de la niñez. En general, la participación infantil se limita al espacio de organizaciones y proyectos sociales en sectores de escasos recursos con apoyo de la cooperación internacional (Aróstegui \& Quintana, 2005).

\section{Relaciones con pares}

Aparte del ámbito escolar, encontramos pocas referencias en los estudios analizados sobre los espacios de interrelación entre niños/as. En un estudio realizado en 160 familias de 4 municipios se observó que las interacciones de los niños/as y adolescentes en su familia se producen, sobre todo, con hermanas/hermanos, madres y sobrinos/primos. A partir de los 6 años, los niños/as se vuelven más independientes de sus padres/madres y salen a jugar con vecinos (Castillo \& Serra, 2003).

El diagnóstico realizado con familias de Ciudad Sandino muestra que las actividades recreativas que realizan los niños/as fuera del horario escolar con familiares, amigos o vecinos, están diferenciadas claramente por sexo y tienen el obstáculo de un espacio limitado en sus casas y de falta de parques de recreación seguros y limpios en su comunidad (Zúñiga \& Pineda, 1997).

Todos los estudios muestran la importancia que le dan los niños/as al juego como forma de recreación e interacción social con sus pares, y los juegos están diferenciados por género desde tierna edad. En un estudio en Managua los padres/madres entrevistados reconocen que sus hijos pasan varias horas diariamente frente a la televisión en su mayoría viendo programas violentos. Por otro lado, muchos niños/as participan frecuentemente en actividades religiosas según la afiliación de sus padres/madres (Zúñiga \& Pineda, 1997).

Otro estudio confirma que los lugares públicos de recreación son muy escasos en las comunidades pobres, generalmente existe un solo lugar público de referencia en cada comunidad (Ej. cancha, cuadro, parque) pero a muchos niños/as no les permiten acceder por los riesgos existentes (instalaciones rotas, basura, delincuentes, borrachos y adictos). $\mathrm{Al}$ preguntarles a los niños/as sobre el trato que reciben por parte de los adultos en su 
barrio o comarca, la mitad de los entrevistados afirmaron que éste no es bueno debido a que muchas veces son insultados, les ponen sobrenombres, los golpean y sus necesidades no son atendidas (Castillo \& Serra, 2003).

\section{Educación}

Todos los estudios muestran una alta asistencia de los niños/as a la escuela, expresando que cuentan con apoyo de sus padres/madres, principalmente la madre, mediante la compra de útiles, asistencia a reuniones con maestros, ayuda en las tareas escolares, preparación de uniforme y desayuno. Tanto los padres/madres como los maestros y los niños valoran muy positivamente el acceso a la educación formal. Los pobres la perciben como la esperanza de una vida mejor y los no pobres la valoran para aprender nuevas cosas. Entre las cosas que más valoran los niños/as de la escuela son los maestros, los compañeros y el deporte. Es interesante ver que muchos padres/madres consideran que la responsabilidad principal de la educación recae en las maestras/os, mientras que éstas/os señalan la falta de apoyo de los padres/madres a las labores escolares de sus hijos (Castillo \& Serra, 2003).

Al preguntarles sobre los derechos de los niños/as, el principal derecho que mencionaron los padres/madres de Ciudad Sandino fue el acceso a educación gratuita. En siguiente prioridad señalaron el recibir alimentación y cariño. Casi todos los niños/as declaran asistir a la escuela por interés personal y aspiran en un futuro a ser profesionales o técnicos a pesar de que carecen de condiciones mínimas para estudiar en casa (luz, espacio, mesa, tranquilidad) (CANTERA, 1995).

En un estudio en Managua, más de la mitad de los padres/madres entrevistados dice apoyar a sus hijos diariamente en sus tareas escolares y visitan a la maestra para saber sobre el desempeño de sus hijos una vez al mes o semanalmente. La mayoría dice haber abordado algunos temas de sexualidad con sus hijos/as en su tránsito a la adolescencia (Zúñiga \& Pineda, 1997). En igual sentido, una encuesta a 120 niños de 10-12 años de 4 municipios indicaba que el 72.5\% había recibido algún tipo de educación sexual, más en sectores urbanos y a través de la escuela, sin embargo, un 67\% deseaba recibir información sobre esa temática (Ortega et al., 2000).

La principal razón de abandono escolar es la pobreza familiar en un contexto donde el Estado no asegura la gratuidad de la educación, y los niños/as deben de realizar diversas labores domésticas o de generación de ingresos. El estudio de familias con padres/madres emigrantes realizado en 5 municipios del país muestra que las remesas ayudan a los gastos de educación, alimentación y salud de sus hijos, pero se debilita el control de sus actividades y hay mayores oportunidades de "callejisarse". Sólo un 42\% de los niños/as entrevistados reconoce que sus responsables (madres, abuelas, tías) les ayudan siempre en sus tareas escolares, quedando el 58\% que también asiste a la escuela sin contar con apoyo en su hogar (PDH, 2004). 


\section{Normación, disciplina y decisiones}

Los estudios muestran que la mayoría de los padres/madres no considera a los niños como sujetos de derechos con capacidad de opinión y de decisión, sino como personas que hay que cuidar y proteger. La mayoría de los padres/madres encuestados a nivel nacional considera que su hijo/a tiene un buen comportamiento cuando "obedece", "está contento" y "no molesta", caso contrario toman medidas disciplinarias (Zúñiga \& Pineda, 1997). Por su parte, los niños reconocen la autoridad paterna. En un estudio con 120 niños (10-14 años) un 98\% estaba de acuerdo en que "se debe siempre amar, respetar y obedecer a los padres", pero pide que los tomen en cuenta. (Ortega et al., 2000:43).

En una encuesta nacional, un 45\% de los niños/as dice que los adultos siempre los tienen en cuenta en las decisiones de su casa (más los varones), un 39\% dice que algunas veces y el 14\% que nunca lo hacen (PDH, 2002). Por otro lado, en un diagnóstico en Ciudad Sandino la mayoría de los niños/as entrevistados dice no ser tomado en cuenta en las decisiones importantes de su hogar. Sin embargo, un grupo reconoce que sus padres/madres les permiten expresar sus opiniones y sentimientos (CANTERA, 1995).

Un estudio realizado en 66 barrios de Managua trata de precisar cuáles son los aspectos de la vida cotidiana de los niños/as en los que ellos/as tienen posibilidad de decidir y encuentra que existe una autonomía en aspectos tales como la elección de amistades, los juguetes, el peinado, la ropa, y qué hacer en el tiempo libre. Por su parte, los padres/madres señalan que un punto de conflicto frecuente con sus hijos es sobre las amistades que han elegido. En caso de tener alguna necesidad o solicitar un permiso, la mayoría de los niños/as acude a su madre, con quien tienen una relación de comunicación más estrecha, y es quien toma la mayoría de las decisiones sobre la vida cotidiana de sus hijos/as (permisos, qué comprar, dónde salir, castigos) (Agudelo et al., 2000).

Todos los estudios coinciden en destacar el uso de métodos drásticos de corrección por parte de los adultos cuando consideran que los niños/as han cometido alguna falta, que es básicamente desobedecer una orden paterna o materna. Un estudio realizado en 160 hogares muestra que la mitad de los adultos encuestados reconoce el uso de formas violentas (51.8\%) tales como gritarle y pegarle a sus hijos/as, ${ }^{1}$ en menor medida acuden al diálogo y la reflexión (37\%), mientras que otros padres/madres prefieren castigarlos prohibiéndoles el uso de algún bien (Ej. televisión) o la realización de alguna actividad (Ej. jugar) (Castillo \& Serra, 2003).

En cuanto a las diferencias de género, un estudio realizado en Managua señala que el castigo físico y los gritos son formas usuales de "corregir a los hijos", en mayor medida a los varones, mientras que en el caso de las mujeres usan más la explicación y las prohibiciones (Zúñiga \& Pineda, 1997). En cuanto a diferencias socio-económicas, otro estudio indica que la violencia intrafamiliar es mayor en sectores pobres que no pobres, en dos tercios de los niños/as pobres aparecen conflictos con algún familiar (tío, hermano, padre o padrastro) quien le ha causado maltratos físicos o psicológicos (Pineda \& Guerra, 1997). Esta situación de violencia intrafamiliar se asocia con la escasez de recursos para garantizar la comida diaria, y las condiciones de hacinamiento y promiscuidad donde convive un conjunto de 
familias viviendo bajo un mismo techo (Fauné, 1995b).

Varios estudios muestran en el tema de castigos una contradicción entre la opinión de los niños y la de sus padres/madres, así como entre la declaración verbal de éstos, que recoge el discurso de los derechos humanos, y la realidad observada de maltrato físico y psicológico. Así el diagnostico realizado en Ciudad Sandino recoge la opinión de 100 niños/as, de los cuales $45 \%$ dice que recibe castigo físico (cinturón, mecate, vara) y en menor medida prohibiciones (27\% ver televisión, jugar, pasear) y regaños (11\%). Esto contrasta con la opinión de los padres/madres que dicen usar generalmente el llamado de atención verbal (64\%) o las prohibiciones (20\%). El tema del castigo es motivo frecuente de desacuerdos entre padres, madres o tutores, según reconocen los adultos encuestados (CANTERA, 1995).

Una encuesta nacional a 1,200 hombres encuentra que la mayoría (53\%) considera que un buen padre debe castigar físicamente a sus hijos cuando se portan mal para corregirlos. Sin embargo, un $42.3 \%$ de los hombre encuestados opina lo contrario y un $96 \%$ piensa que un padre debe ser cariñoso con sus hijos (Centeno \& Castillo, 2005). En otro estudio, al preguntarles a los niños/as cómo les gustaría que se comportaran sus padres/madres, ellos dijeron: "que fueran cariñosos, amables, buenos", "tranquilos, comprensivos", "nos den consejo", "confíen en nosotros", "que no nos peguen ni maltraten". Es decir, que encontramos respuestas que aluden por contraposición al duro régimen disciplinario existente (Castillo \& Serra, 2003:80).

En Nicaragua se reportan a diario casos de maltrato físico, abusos sexuales y violencia contra las mujeres y los niños/as. En muchos casos los abusadores son hombres conocidos y allegados a la familia: padres/madres, convivientes, ex convivientes, hermanos, hermanastros. El maltrato trae consecuencias en la niñez, además de las lesiones físicas provoca temor, agresividad, timidez, fuga del hogar.

\section{Rupturas y conflictos}

En general los grupos familiares son inestables por diversas razones: muchas parejas se basan en uniones de hecho no formales; la cultura predominante del machismo que "naturaliza" la poligamia y la irresponsabilidad paterna; las migraciones de padre/madre en búsqueda de fuentes de ingreso (incluye maquilas); en zonas rurales, una parte importante de las familias no se constituyen sobre la base del acuerdo de voluntades entre la pareja, sino por "el rapto" o la entrega paterna de la novia.

Un estudio realizado en Managua señala una ruptura matrimonial frecuente, un 49\% de los hombres entrevistados que tenían entre 15 y 40 años se había separado de su matrimonio inicial y había formado una nueva relación de pareja; en menor medida (32\%) las mujeres habían tenido una experiencia similar (Montenegro, 2000). La ruptura familiar afecta seriamente las relaciones padres/madres-hijos y la crianza de éstos, especialmente en el caso de la atención de los papás a sus hijos/as (Montenegro, 2000).

Se estimaba en 220,000 los niños/as que no vivían con su papá y/o su mamá en el año 2000, encontrándose en hogares con parientes o con padres adoptivos, en la calle, o en los escasos 
centros de atención que tienen ONG y el Ministerio de la Familia. El perfil de la familia de estos niños se caracteriza por el abandono paterno o materno, hijos no deseados, madre sola en extrema pobreza, maltrato a niños/as por un padrastro o una madrastra, presencia de alcoholismo y violencia, abuela incapaz de atenderlos (PNUD, 2000).

Un motivo de ruptura familiar es la violencia. Una de cada tres mujeres ha sufrido alguna vez violencia física o sexual por parte de su esposo o compañero, lo cual acarrea lesiones físicas y psíquicas. La mayoría de las mujeres maltratadas no lo reporta a ninguna institución y un tercio de las mujeres encuestadas considera que el uso de la violencia se justifica en caso de infidelidad, descuido del hogar y de los hijos, y salida sin el permiso del esposo o compañero. Algunos estudios realizados en Nicaragua muestran que la violencia intrafamiliar se repite de una generación a la siguiente, es decir, que quienes han sido maltratados en su niñez actúan de igual forma con sus hijos/as. Esta problemática de la violencia afecta la salud y las relaciones sociales, también es un obstáculo para la democracia y el desarrollo humano (PNUD, 2000).

\section{Trabajo infantil}

Los estudios indican que la mayoría de los niños/as realiza tareas principalmente en su hogar: limpiar, barrer, lavar platos y ropa, planchar, acarrear leña y agua, hacer compras, cocinar, atender la huerta o el negocio familiar. Las tareas aumentan con la pobreza de la familia, la edad de los niños/as y el sexo, ya que recaen más sobre las mujeres dentro de una división tacita del trabajo: al varón le tocan más tareas en los espacios públicos (Ej. mandados), a las niñas le corresponden labores domésticas en preparación para su rol de madre.

Un diagnóstico de Managua identifica la distribución de labores domesticas mostrando una carga considerable en la madre (54\%) seguida por las hijas (19\%), luego el padre (16\%) y los hijos (11\%) (Zuñíga, 1997). Estos datos se confirman en otros estudios, es decir, que las niñas colaboran en mayor medida que los varones en tareas tales como limpiar la casa, cuidar hermanos, ayudar en la cocina, lavar y planchar (Agudelo et al., 2000). Esto indica que se reproduce la tradicional división del trabajo por género que asigna a las mujeres el ámbito domestico y a los hombres el ámbito público. Sin embargo, la mayor participación de las mujeres en actividades de generación de ingresos fuera del hogar no ha modificado esa distribución y por tanto las mujeres sufren de doble o triple jornada laboral al asumir el rol de proveedoras del sustento económico de las familias y la jefatura del hogar (Centeno \& Castillo, 2005).

Se ha observado que la mayoría de las mujeres de sectores pobres que trabajan por cuenta propia utiliza la casa como lugar de trabajo, lo que agrava el problema de hacinamiento de la familia y la dificultad para las mujeres de realizar tareas domésticas y productivas al mismo tiempo y en la misma casa. Además, en situación de pobreza la mujer madre debe de administrar el raquítico ingreso familiar vía la reducción de comida en cantidad y calidad, y también de combustible, luz, agua, vestimenta y gastos no básicos (Fauné, 1995c). 
y que le gusta realizar estas labores porque ayudan a su familia. Por el contrario, el recargo de tareas domésticas es criticado especialmente por niñas púberes y adolescentes ya que limita su posibilidad de estudio y recreación, a diferencia de los varones de igual edad (Castillo \& Serra, 2003).

En relación al trabajo para generación de ingresos, los estudios realizados confirman la participación, de forma creciente con la edad, de niños/as de familias pobres en distintas labores que son remuneradas monetariamente a sus padres/madres o tutores. Asimismo, muchos padres/madres pobres opinan que sus hijos deben contribuir al mantenimiento económico de la familia y algunos le dan más importancia a esto que a la asistencia a la escuela (PDH, 2002).

Un estudio realizado en Managua muestra que la mayoría de los niños pobres realiza actividades remuneradas en la calle con un promedio de 3 a 6 horas diarias, y que iniciaron entre los 6 y los 8 años. Los niños/as entrevistados tienen una percepción ambivalente respecto al trabajo remunerado. Por un lado destacan que ayudan a su familia, se sienten útiles, pueden estudiar y tienen ingresos; pero, por otro lado, señalan aspectos negativos como que nos les queda tiempo para estudiar y jugar, los maltratan en la calle y los explotan en el trabajo (Pineda \& Guerra, 1997). Este fenómeno es observable a nivel centroamericano, y según Fauné (1995c:27), quien destaca la triste realidad para la niñez pobre, "la incorporación temprana al trabajo reduce abruptamente la niñez. La adolescencia no alcanza a iniciarse cuando termina con el emparejamiento y fecundidad precoz”.

\section{Conclusiones}

Existe una serie de valiosos estudios realizados en Nicaragua en los últimos 10 años que arrojan luces sobre los procesos de crianza de la niñez en el ámbito familiar. Ellos enfatizan en las percepciones sobre las prácticas de crianza y se enfocan en familias de escasos recursos que habitan en espacios urbanos. Sin embargo, considerando el rol clave que juega la familia en la sociedad actual y la problemática que afecta la crianza de la niñez, se podría concluir que los esfuerzos investigativos realizados hasta la fecha para profundizar el análisis de esta temática han sido más bien reducidos.

En los estudios consultados, la crianza de hijos/as se aborda teóricamente como un proceso de socialización primaria, es decir, como transmisión de valores, concepciones y actitudes propias de la cultura dominante al mismo tiempo que se desarrollan las capacidades de los niños conforme su edad y las condiciones socio-económicas de la familia. Sin embargo, no se trata de un proceso unidireccional y mecánico, sino que la crianza implica una interacción entre niños/as y adultos. Por otro lado, la niñez no es considerada como una realidad uniforme y universal, sino variable conforme su mundo social y cultural. La crianza abarca componentes ideales (creencias, saberes, valores) y prácticos (acciones habituales). Ambos elementos interrelacionados configuran "sistemas de crianza".

Los resultados confirman que las madres juegan un rol insustituible en la atención a los hijos/as tanto en el ámbito afectivo como de sus necesidades básicas; también señalan el rol de los hermanos y hermanas en el cuido de sus hermanos/as pequeños y apoyo en 
tareas escolares. La responsabilidad materna es aún mayor en los hogares bajo jefatura femenina.

Las investigaciones identifican una contradicción entre discurso y práctica de crianza. Así, muchos varones opinan que la crianza de los hijos es una labor compartida entre padre y madre, aunque en la práctica se constata que las mujeres suelen asumir las labores cotidianas como el cuido, la alimentación y el apoyo escolar. Esta actitud se asocia con la concepción de paternidad tradicional predominante entre los hombres nicaragüenses, que considera al padre como el proveedor económico y a la madre como la responsable de la crianza de los hijos/as. No obstante, vale la pena destacar que se observa un perfil creciente de padres con una mentalidad de compartir la crianza con las madres y brindar afecto a sus hijos, además de apoyo económico.

Los estudios seleccionados muestran en Nicaragua una ambivalencia de la vida familiar y de estilos de crianza. Por un lado, la familia constituye la principal organización de supervivencia material y afectiva basada en la ayuda recíproca que se establece entre parientes; por otro lado, representa un espacio de socialización de relaciones de poder y de violencia que fomentan actitudes de dominación y de sumisión. El hogar puede convertirse en un espacio de injusticia, particularmente contra las mujeres y las niñas, obstaculizando un desarrollo humano integral. Para enfrentar esta problemática, no es suficiente la aprobación de leyes, sino que éstas deben de complementarse con acciones dirigidas al cambio de patrones culturales tan arraigados en el imaginario colectivo.

Es preocupante observar que la mayoría de los padres/madres no considera a los niños como sujetos de derechos con capacidad de opinión y de decisión, sino como personas que hay que cuidar y proteger. Por tanto, el ámbito de decisiones que tienen los niños es mínimo y está vinculado a aspectos tales como su ropa, juegos, amistades, tiempo libre. La mayoría de los niños/as acude a su madre, quien toma la mayoría de las decisiones sobre las actividades cotidianas de sus hijos/as.

Es habitual el uso de métodos drásticos de corrección por parte de los adultos cuando consideran que los niños/as han cometido alguna falta, básicamente desobedecer una orden paterna o materna. Los castigos físicos y el maltrato verbal son justificados por muchos padres/madres con el fin de "corregir" a sus hijos/as, aunque varios estudios muestran una contradicción entre la opinión de los niños sobre el maltrato que reciben y la de sus padres/ madres.

La educación escolar es valorada positivamente por todos los niños/as y los padres/madres como un medio de aprendizaje, de interacción con pares y de ascenso social. Las diferencias socio-económicas son claves en el acceso a una educación de calidad. En las familias de mayores recursos, los hijos tienen acceso a una formación técnica o profesional en centros privados de calidad, en cambio los hijos de familias de escasos recursos deben acudir a los centros públicos con escasos medios didácticos y con profesores mal pagados, o quedan fuera del sistema porque sus familias no pueden costearles los estudios y más bien los envían a trabajar para generar ingresos. 
Los estudios indican que la mayoría de los niños/as realiza tareas en su hogar, mayormente en familias pobres, y éstas recaen más sobre las niñas en preparación para su rol de madre. Por otro lado, observamos un sector considerable de niños/as que trabajan en actividades de generación de ingresos familiares de forma creciente con la edad y en muchos casos, en labores que ponen en riesgo su salud y su educación.

En las últimas décadas se observan diversas experiencias de participación infantil en espacios públicos a través de creaciones artísticas, encuentros y planteamientos que promueven organizaciones civiles abocadas a la promoción de los derechos de la niñez. Sin embargo, resta un largo camino para extender la participación efectiva de los niños/as en el ámbito familiar, escolar y laboral en consonancia con la visión de sujetos de derecho contemplada en el marco legal vigente.

Los estudios identifican dos modelos contrapuestos de autoridad y crianza: por un lado, un modelo autoritario caracterizado por la imposición arbitraria, la comunicación unilateral y la violencia contra los más débiles (niños, mujeres), que tradicionalmente ha predominado en Nicaragua, y por el otro, un modelo democrático en gestación donde predomina el consenso, la negociación y la comunicación bilateral en las decisiones familiares, lo cual desarrolla en los niños/as habilidades de comunicación y negociación, y estimula la autonomía, la conciencia critica y el respeto a los derechos de los demás. Mientras el primer modelo ha sido estudiado en sus manifestaciones prácticas en Nicaragua, se carece de investigaciones que revelen el funcionamiento y viabilidad del modelo participativo.

Gran parte de los estudios hechos en Nicaragua son diagnósticos focalizados en un territorio local en preparación de una intervención social con la niñez. Estos estudios enfrentan el difícil reto de, por un lado, comprender la compleja dinámica social con sus múltiples dimensiones a través de escasas fuentes de información y en corto plazo, y por otro lado, plantear propuestas factibles y pertinentes para superar las limitaciones encontradas.

Desde el punto de vista metodológico, algunos estudios muestran valiosos resultados producto de la combinación creativa de técnicas cuantitativas y cualitativas que permiten revelar tanto las representaciones sobre crianza infantil como las prácticas en el ámbito familiar. En los estudios basados en encuestas a nivel nacional encontramos resultados representativos de situaciones y tendencias amplias que nos permiten comparar los estilos de crianza entre distintas situaciones familiares (rural-urbano, pobre-no pobre). Sin embargo, el uso de cuestionarios con niños y niñas de 6 a 12 años es de validez debatible dadas las brechas de conceptualización entre adultos y niños, especialmente en encuestas cerradas y aplicadas al azar en hogares donde no existía una confianza previa con el encuestador. Queda pendiente el reto de profundizar los estudios interdisciplinarios, explicativos y comparativos entre estilos de crianza por sexo y edad (hijos/as y padres/madres), entre hogares con jefe mujer y hogares con jefe varón, entre familias de clases socio-económicas distintas, entre residentes urbanos y rurales, y entre diferentes grupos étnicos del país. Además queda el desafío de realizar estudios con una metodología participativa que incluya la perspectiva de los niños/as y adolescentes tanto en el análisis de la problemática como en las propuestas de alternativas para avanzar un modelo de crianza respetuoso de los derechos humanos y promotor de relaciones sociales justas y solidarias. 


\section{Agradecimientos}

Agradezco a Manuel Ortega, Osvaldo Montoya y Manfred Liebel por sus valiosas observaciones al borrador del artículo. Próximamente se publicarán los resultados del trabajo de campo realizado en Nicaragua y en seis países de América Latina.

\section{Notas}

1 El castigo físico se realiza usando el cinturón, el mecate, el azote, la tajona y la vara.

\section{Referencias bibliográficas}

AGUDELO I., MONTENEGRO S. \& OBREGÓN R. (2000). Las representaciones filiales y parentales sobre las relaciones en la familia. CINCO-Red Barna Noruega: Managua. Mimeo.

AMAYA, L. (2004). La situación de la niñez en Nicaragua. INPRHU: Managua.

ARÓSTEGUI, N. \& QUINTANA, M.E. (2005) La Participación infantil en Nicaragua. Save the Children Noruega: Managua.

CANTERA (1995). La incidencia de la familia en la formación de la infancia. Managua. Mimeo.

CASTILLO, M. \& SERRA, L. (2003). Diagnóstico sobre niñez y adolescencia en Nicaragua. UCA-PLAN Internacional: Managua.

CENTENO, R. \& CASTILLO, M. (2005). Masculinidad y factores socioculturales asociados a la paternidad en Nicaragua. CASC-UCA/CEPAL: Managua.

CHAMORRO RUÍZ, M. \& GUTIÉRREZ BACA, S. (2001). "Nicaragua: la niñez y la adolescencia en la estrategia reforzada de reducción de la pobreza". En Informe de Década sobre Niñez y Adolescencia. Consejo Nacional de Protección y Atención Integral a la Niñez y Adolescencia CONAPINA: Managua.

COORDINADORA DE ORGANIZACIONES DE NIÑEZ Y ADOLESCENCIA, CODENI. (2003). Sistematización de metodologías de promoción de participación de niñas, niños y adolescentes trabajadores. CODENI: Managua.

COREA TÓRREZ, N. et al. (2000). Estudio sobre las prácticas de crianza de la niñez nicaragüense. PAININ-CONAPINA: Managua. Mimeo.

FAUNÉ, M.A. (1995a). "Las familias, las mujeres: la realidad". En Envío, Revista de la Universidad Centroamericana, $\mathrm{N}^{0} 160$. UCA:Managua. Disponible en: www.envio.org.ni. (1995b). "Hogares ampliados y en manos de las mujeres". En Envío, Revista de la Universidad Centroamericana, $N^{0} 161$. UCA: Managua. Disponible en: www.envio.org. ni.

_(1995c). "Familias: violencia y sobrevivencia". En Envío, Revista de la Universidad Centroamericana. $\mathrm{N}^{\circ} 162$. UCA: Managua. Disponible en: www.envio.org.ni.

GORDILLO, A.et al. (2004). Violencia contra la niñez y la adolescencia. Plan Internacional: Managua.

Instituto Nicaragüense de Estadísticas y Censos, (INEC). (2001). Indicadores Sociales de la Niñez Nicaragüense: Managua.

(2002). Encuesta Nicaragüense de Demografía y Salud 2001: Managua. 
(2006). Censo Nacional de Población y Vivienda 2005: Managua.

MITRAB-OIT. (2007). Encuesta Nacional de Trabajo Infantil y Adolescente en Nicaragua ENTIA 2005: Managua.

MONTENEGRO, S. (2000). La cultura sexual en Nicaragua. CINCO: Managua.

MONTOYA, O. (2001). Educación reproductiva y paternidad responsable en Nicaragua. CEPAL: Nicaragua.

ORTEGA, M. et al. (2000). El embarazo en adolescentes en Nicaragua: una aproximación sociocultural. CASC-UCA: Managua.

PINEDA, G. \& GUERRA, B. R. (1997). Cómo los niños y niñas ven su mundo. Redd Barna Noruega: Managua. Mimeo.

PROCURADURÍA DE DERECHOS HUMANOS (PDH). (2002). Así vemos, así queremos Nicaragua. La percepción que tiene la niñez y la adolescencia de su entorno social, político, económico y persona. PDH: Managua.

_ (2004). Distancia sin olvido. Investigación socio-jurídica sobre la emigración de madres y padres, y los efectos en sus hijas e hijos. Managua. Mimeo.

PROGRAMA DE NACIONES UNIDAS PARA EL DESARROLLO. (2000). Informe de Desarrollo Humano de Nicaragua. PNUD: Managua. (2005). Informe de Desarrollo Humano. PNUD: Washington.

ZÚNIIGA, S. \& PINEDA, G. (1997). Estudio sobre prácticas de crianza en el barrio B-15 del Distrito 6 de Managua. CAPRI: Managua. Mimeo. 